\title{
How Fibrinolysis was defeated by PCI
}

\author{
Victor Gurewich MD \\ Professor of Medicine, Harvard Medical School.
}

Corresponding Author: Victor Gurewich, Professor of Medicine, Harvard Medical School.

\section{Received Date: 11 September 2021 | Accepted Date: 01 October 2021 | Published Date: 07 October 2021}

Citation: V Gurewich. (2021) How Fibrinolysis was defeated by PCI. Journal of Clinical and Laboratory Research. 3(4); DOI: 10.31579/27680487/049

Copyright: (C) 2021 Victor Gurewich. This is an open-access article distributed under the terms of the Creative Commons Attribution License, which permits unrestricted use, distribution, and reproduction in any medium, provided the original author and source are credited.

Fibrinolysis refers to the natural enzymatic system responsible for dissolving a blood clot or thrombus. Since an intravascular thrombus is responsible for almost all heart attacks and most strokes, interest in fibrinolysis, the only medical treatment, dominated medical interest in the 60 's-90's. Since that time, the incidence of heart attacks and strokes has not changed much, but interest in fibrinolysis has faded. Instead, interest has shifted to catheter removal of clots, called percutaneous coronary intervention (PCI) which is a time-consuming that delays reperfusion considerably, and is much more costly than fibrinolysis. It is also a cruder treatment that can only remove clots larger than the catheter, but it is very well reimbursed.

By contrast, fibrinolysis is a much faster, cheaper, treatment which is not limited to larger vessels, so what happened to it? The fault is both intellectual, related to a misunderstanding of fibrinolysis, and financial or profit motivated. The latter is the easiest to identify. The current treatment of a heart attack or stroke is PCI, which is well-compensated and has become an important revenue source for cardiology departments and hospitals. It has become the favorite treatment also because fibrinolysis was conveniently misunderstood.

For more than three decades, fibrinolysis has meant the administering a single fibrinolytic, usually tissue plasminogen activator (tPA), which is known to be a weak fibrinolytic. More importantly, it has been recognized since 1984, when prouPA was discovered [1] that fibrinolysis requires two fibrinolytics, tPA and prouPA, which is much more effective than and tPA alone. In fact, prouPA (which has two forms), is responsible for two-thirds of fibrinolysis and tPA one third. This ratio is also consistent with gene deletion studies in animals.

These findings describing the presence and importance of the second activator were ignored, and fibrinolysis continued to mean giving tPA alone. This meant deliberately handicapping fibrinolysis which provided an excuse to change reperfusion treatment of heart attack and, when possible, stroke to a catheter, PCI). This is a more financially rewarding treatment, but it also denies the natural system of dissolving blood clots, which requires two fibrinolytics [2]. By undermining fibrinolysis it left PCI to become the treatment of choice for heart attacks and some strokes, and is a treatment that was encouraged more by its generous reimbursement than by its efficacy. The evidence for this is that PCI to date has never been compared with fibrinolysis. It was only compared with "fibrinolysis" by tPA which is one third of fibrinolysis. The latter requires both $\mathrm{PPA}$ and prouPA. Comparing PCI with fully effective fibrinolysis has so far been avoided, leaving PCI as the treatment of choice, and a major source of revenue but not supported by any study of its efficacy.

Fibrinolysis by the tPA and uPA combination, which promises to be the most effective method to remove an obstructive intravascular blood clot has been successfully ignored, and fibrinolysis with tPA alone has been retained providing an excuse for PCI.

The catheter brings in more money, but is less effective and is associated with a small risk, whereas fibrinolysis by both IPA and prouPA is the most effective treatment of heart attack and stroke and carries little to no bleeding risk, since only a $5 \mathrm{mg}$ bolus of tPA is required.

\section{References:}

1. Gurewich V, Pannell R, Louie S, Kelley P, Suddith RL, Greenlee R. (1984). Effective and fibrin-specific clot lysis by a zymogen precursor form of urokinase (pro-urokinase). A study in vitro and in two animal species. J Clin Invest. 73:1731-1739.

2. Pannell R, Li S, Gurewich V. (2015). Highly effective fibrinolysis by a sequential synergistic combination of mini-dose tPA plus lowdose mutant proUK. PLOS One. 10:1-15. 